\title{
Modeling of epoxy dispensing process using a hybrid fuzzy regression
}

\author{
approach \\ K.Y. Chan ${ }^{1}$ and C.K. Kwong ${ }^{2}$ \\ ${ }^{1}$ Department of Electrical and Computer Engineering, \\ Curtin University, \\ Australia \\ ${ }^{2}$ Department of Industrial and Systems Engineering, \\ The Hong Kong Polytechnic University, \\ Hong Kong, PRC
}

\begin{abstract}
In the semiconductor manufacturing industry, epoxy dispensing is a popular process commonly used in die-bonding as well as in microchip encapsulation for electronic packaging. Modelling the epoxy dispensing process is important because it enables us to understand the process behaviour, as well as determine the optimum operating conditions of the process for a high yield, low cost and robust operation. Previous studies of epoxy dispensing have mainly focused on the development of analytical models. However, an analytical model for epoxy dispensing is difficult to develop, because of its complex behaviour and high degree of uncertainty associated with the process in a real world environment. Previous studies of modelling the epoxy dispensing process have not addressed the development of explicit models involving high order and interaction terms, as well as fuzziness between process parameters. In this paper, a hybrid fuzzy regression (HFR) method integrating fuzzy regression with genetic programming is proposed to make up the deficiency. Two process models are generated for the two quality characteristics of the process, encapsulation weight and encapsulation thickness based on the HFR, respectively. Validation
\end{abstract}


tests are performed. The performance of the models developed based on the HFR outperforms the performance of those based on statistical regression and fuzzy regression.

Keywords: evolutionary computation, fuzzy regression, genetic programming, epoxy dispensing, microchip encapsulation, electronic packaging, process modelling, semiconductor manufacturing

\section{Introduction}

Epoxy dispensing is a manufacturing process by which fluid materials are delivered to substrates, boards or work-pieces in a controlled manner. This process has been widely used in various packaging processes in the electronics and semiconductor manufacturing industry such as integrated circuit (IC) encapsulation, die-bonding and surface mount technology [1]. In today's competitive market, the variables of this manufacturing process need to be controlled at each of the many processing steps in the manufacturing line. The process directly affects the overall quality of the finished product, as well as the throughput of the production line. All the variables controlling the desired outputs in a given process need to be understood and optimized for tight control. However, epoxy dispensing is a highly non-linear process and creates a highly coupled multi-variable system involving extremely complex inter-relationships among the epoxy properties, process conditions, needle design parameters and overall encapsulation quality [2]. In semiconductor manufacturing, trial-and-error remains a very common method used to identify proper process parameters setting. However, this method involves long process development time and optimum encapsulation quality may not be obtained. Therefore, it is necessary to develop an accurate model for the process.

Analytical models are attractive, as they provide a fundamental understanding of the relationships between the various input and output parameters. Various analytical models for modelling the epoxy dispensing process for electronic packaging have been developed by Chen [1], Li et al. [3], and Chen and Ke [2]. Relationships between process variables and 
process responses are represented by a set of governing partial differential equations, in which behaviors of the epoxy dispensing process can be analyzed. A comparative study of modelling epoxy dispensing process using computational epoxy dynamics has also been carried out Hong and Li [4]. Theoretical investigations of epoxy dispensing processes have been studied by Li and Deng [5] and Chen [6]. However, an analytical model for epoxy dispensing, which can provide accurate results, is very difficult to develop, as the behaviours of epoxy dispensing are complex and the degree of uncertainty associated with the process in a real world environment is high.

Various modelling approaches have been introduced to develop epoxy dispensing models based on experimental data. Statistical regression, neural networks [7] and fuzzy neural networks [8] have been employed for modelling epoxy dispensing processes. Statistical regression models are accurate over the range in which they are developed. As a result, statistical regression models can be applied only if the given data are distributed according to a statistical model, and the relationship between dependent and independent variables is crisp. Neural networks and fuzzy neural networks have been adopted to develop process models for epoxy dispensing, where genetic algorithms have been employed for network training. Both approaches have the capability of modelling nonlinear, complex and noisy processes. However, a large number of experimental datasets is normally required to develop process models using these two approaches, neural networks and fuzzy neural networks. It is usually not available in epoxy dispensing processes, as the time taken in conducting experiments in real-world manufacturing industries is limited. On the other hand, the epoxy dispensing models are developed within a black box structure based on these two approaches, which lacks transparency. Therefore, these two approaches are not appreciated by manufacturing engineers in developing epoxy dispensing processes.

Fuzzy linear regression [9] has the distinct advantage that a manufacturing process, which has a high degree of fuzziness, can be modeled using few, or even incomplete 
experimental datasets [10-11]. Ip et al. [12] introduced the fuzzy linear regression to develop process models for epoxy dispensing processes. However, all interaction and high order terms in epoxy dispensing processes were not considered by the fuzzy linear regression. In fact, interaction effect and non-linearity exist in the epoxy dispensing processes [2]. Genetic programming (GP) is an evolutionary method which can be used to generate models having interaction and high order terms [13, 14]. For example, Lakshminarayanan et al [15] has applied GP in modeling a nonlinear chemical system with a small amount of data sets, which is usually the case in the real-world industry of process design. Brezocnik and L. Gusel [16] has proposed a GP approach to model radial stress distribution in cold-formed material and the resulting model can be used widely in metal-forming industry. Kok et al. [17] has applied GP for modeling surface roughness in adrasive waterjet machining particle in which satisfactory results can be obtained. Madar et al. [18] have demonstrated using GP to generate a structure of nonlinear models and have employed the nonlinear least square algorithm to perform the associated parameter estimation in the nonlinear models. However, in epoxy dispensing processes, uncertainty due to fuzziness often exists. Therefore, the above GP based methods may not yield the best modeling results in the epoxy dispensing process as those methods neglect the fuzziness.

Therefore, the above existing methods cannot address the whole issues in development of epoxy dispensing models: i) explicit epoxy dispensing models are required by manufacturing engineers; ii) epoxy dispensing processes are highly nonlinear; iii) the collected experimental data is fuzzy nature. In this paper, a hybrid modeling approach integrating genetic programming with fuzzy regression is proposed in order to address the whole issues of development of epoxy dispensing models. We call the approach the hybrid fuzzy regression (HFR). In the proposed approach, the general outcomes of GP are used to construct the structures of nonlinear models based on a tree representation. Based on the tree representation, the structure of the model in polynomial form, which includes interaction and 
high order terms, can be identified. Then, the fuzzy regression method is used to determine the contributions of the branches of the tree. In other words, fuzzy coefficients for all interaction and high order terms of the epoxy dispensing model can be determined. Therefore, the resulting epoxy dispensing models generated by the HFR are explicit, as they are in fuzzy polynomial form. Also, nonlinearity of epoxy dispensing processes can be addressed. Fuzziness of the epoxy dispensing process due to inexact knowledge, which includes human errors in conducting experiments and uncontrollable environment factors involved in the epoxy dispensing process, can be addressed. To study the effectiveness of the proposed HFR in modeling the epoxy dispensing processes, results of the modeling are compared with those based on the commonly used approaches, fuzzy linear regression and statistical regression.

\section{Epoxy dispensing and its modeling}

In a typical epoxy dispensing process for microchip encapsulation, silicon chips are covered using an X-Y numerically controlled dispensing system that delivers the epoxy encapsulant through a needle. The material is commonly dispensed in a pattern, working from the center outward. A fluid dam around the die site and second wire bond points can be made to contain the flow of the material and to produce a more uniform part, as shown in Figure 1.

With the assistance from the company supporting this research [19], a number of experiments to examine the significance of individual parameters for the epoxy dispensing process have been performed. The three individual parameters, compressed air pressure (1 bar to 4 bar) $x_{1}$, pump motor speed (400 rpm to $1000 \mathrm{rpm}$ ) $x_{2}$, and the height between the substrate and the needle (250 to 2,000 steps of the stepping motor) $x_{3}$, are identified as the significant process parameters regarding the epoxy dispensing process by the supporting company. The two main quality characteristics have been identified as encapsulation weight (mg) $y$ and encapsulation thickness $(\mathrm{mm}) z$. The process parameters are considered as the 
significant ones for epoxy dispensing machines, which can be adjusted by engineers to control the qualities of the epoxy encapsulation. Therefore, the three process parameters were recommended to be studied by the supporting company.

Other environmental factors such as environment temperature and fluid viscosity of epoxy are also significant on affecting the qualities of the epoxy encapsulation. Environment temperature normally is set as a constant value in a production shop floor for epoxy dispensing but the temperature may have some fluctuation due to various reasons. In this research, the temperature fluctuation is treated as a source of fuzziness of the process. Fluid viscosity of epoxy resin changes with time during the process of epoxy dispensing. It is also affected by other various factors such as properties of epoxy resin, standby time of epoxy resin, air pressure and volume of epoxy left in the syringes of epoxy dispensing machines at a particular time. Studies of the relationships between fluid viscosity and/or flow rate of epoxy resin and those factors as well as their effects on qualities of epoxy encapsulation involves substantial efforts of experiments and lengthy studies. As the supporting company aimed at developing process models that help engineers to determine proper process parameter settings of epoxy dispensing machines effectively, fluid viscosity of epoxy resin was not considered in this research.

In this research, those uncontrollable factors such as fluctuation of environmental temperature and inconsistent of properties of epoxy resin are considered as fuzziness of the epoxy dispensing process. To deal with the fuzziness, a hybrid fuzzy regression approach is proposed to generate the process models in a form of fuzzy polynomials in which fuzzy coefficients of the process models can be generated to address the fuzziness. 


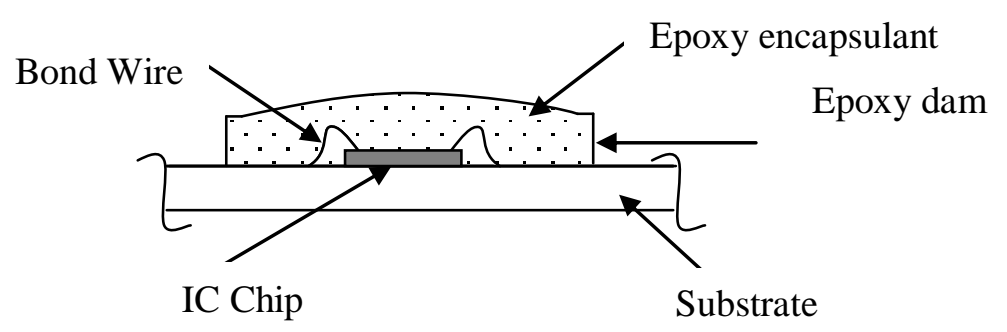

Figure 1 Encapsulation of a chip on board package [12]

Ip et al. [12] have applied fuzzy linear regression [9] to develop process models for the epoxy dispensing as shown in (1).

$$
\tilde{y}=\tilde{A}_{0}+\tilde{A}_{1} x_{1}+\tilde{A}_{2} x_{2}+\tilde{A}_{3} x_{3}
$$

where $x=\left[1, x_{1}, x_{2}, x_{3}\right]^{T}$ is a crisp vector of independent variables of the process model for the epoxy dispensing, and $\tilde{y}$ is the estimated fuzzy output of the process. $\tilde{A}=\left[\tilde{A}_{0}, \widetilde{A}_{1}, \widetilde{A}_{2}, \widetilde{A}_{3}\right]$ is a vector of fuzzy parameters of the epoxy dispensing model. $\tilde{A}_{j}$ is presented in the form of symmetric triangular fuzzy numbers denoted by $\tilde{A}_{j}=\left(\alpha_{j}, c_{j}\right), j=0,1,2,3$.

However, only linear terms of the models can be generated based on the fuzzy linear regression. In fact, the epoxy dispensing process involves the interaction effect of process parameters and non-linear behaviour [2]. It is necessary to consider the interaction effect and non-linearity in the modeling of the epoxy dispensing process so as to generate more accurate process models. The general form [21] of a fuzzy regression model for the epoxy dispensing process, which involves interaction and higher order terms, can be represented as:

$$
\tilde{y}=\tilde{f}_{N R}(x)=\tilde{A}_{0}+\sum_{i_{1}=1}^{3} \tilde{A}_{i_{1}} x_{i_{1}}+\sum_{i_{1}=1}^{3} \sum_{i_{2}=1}^{3} \tilde{A}_{i_{1} i_{2}} x_{i_{1}} x_{i_{2}}+\sum_{i_{1}=1}^{3} \sum_{i_{2}=1}^{3} \sum_{i_{3}=1}^{3} \tilde{A}_{i_{1} i_{2} i_{3}} x_{i_{1}} x_{i_{2}} x_{i_{3}}
$$

where $\tilde{A}_{0}=\left(\alpha_{0}, c_{0}\right), \tilde{A}_{1}=\left(\alpha_{1}, c_{1}\right), \tilde{A}_{2}=\left(\alpha_{2}, c_{2}\right), \tilde{A}_{3}=\left(\alpha_{3}, c_{3}\right), \tilde{A}_{11}=\left(\alpha_{11}, c_{11}\right)$,

$$
\begin{aligned}
& \tilde{A}_{12}=\left(\alpha_{12}, c_{12}\right), \tilde{A}_{13}=\left(\alpha_{13}, c_{13}\right), \ldots \tilde{A}_{33}=\left(\alpha_{33}, c_{33}\right), \tilde{A}_{111}=\left(\alpha_{111}, c_{111}\right), \tilde{A}_{112}=\left(\alpha_{112}, c_{112}\right), \\
& \tilde{A}_{113}=\left(\alpha_{113}, c_{113}\right), \ldots \tilde{A}_{333}=\left(\alpha_{333}, c_{333}\right) .
\end{aligned}
$$


(2) can be rewritten as:

$$
\tilde{y}=\tilde{A}_{0}^{\prime} x_{0}^{\prime}+\tilde{A}_{1}^{\prime} x_{1}^{\prime}+\tilde{A}_{2}^{\prime} x_{2}^{\prime} \ldots \tilde{A}_{40}^{\prime} x_{40}^{\prime}
$$

where the number of terms of (2) and (3) is $40 ; \tilde{A}_{0}^{\prime}=\widetilde{A}_{0}, \widetilde{A}_{1}^{\prime}=\widetilde{A}_{1}, \tilde{A}_{2}^{\prime}=\widetilde{A}_{2}, \ldots \tilde{A}_{40}^{\prime}=\tilde{A}_{333}$; $x_{0}^{\prime}=1, \quad x_{1}^{\prime}=x_{1}, \quad x_{2}^{\prime}=x_{2}, \ldots x_{40}^{\prime}=x_{3} \cdot x_{3} \cdot x_{3} ;$ and $\tilde{A}_{0}^{\prime}=\left(c_{0}, \alpha_{0}\right), \quad \tilde{A}_{1}^{\prime}=\left(c_{1}^{\prime}, \alpha_{1}^{\prime}\right), \ldots$ $\tilde{A}_{40}^{\prime}=\left(c_{40}^{\prime}, \alpha_{40}^{\prime}\right)$. The general form of the fuzzy regression model for the epoxy dispensing process formulated in equation (2) is illustrated in Figure 2, where the solid line represents the center of the fuzzy regression model, the two dash lines represent the fuzziness of the fuzzy regression model and the black dots represent experimental data collected for the epoxy dispensing process.

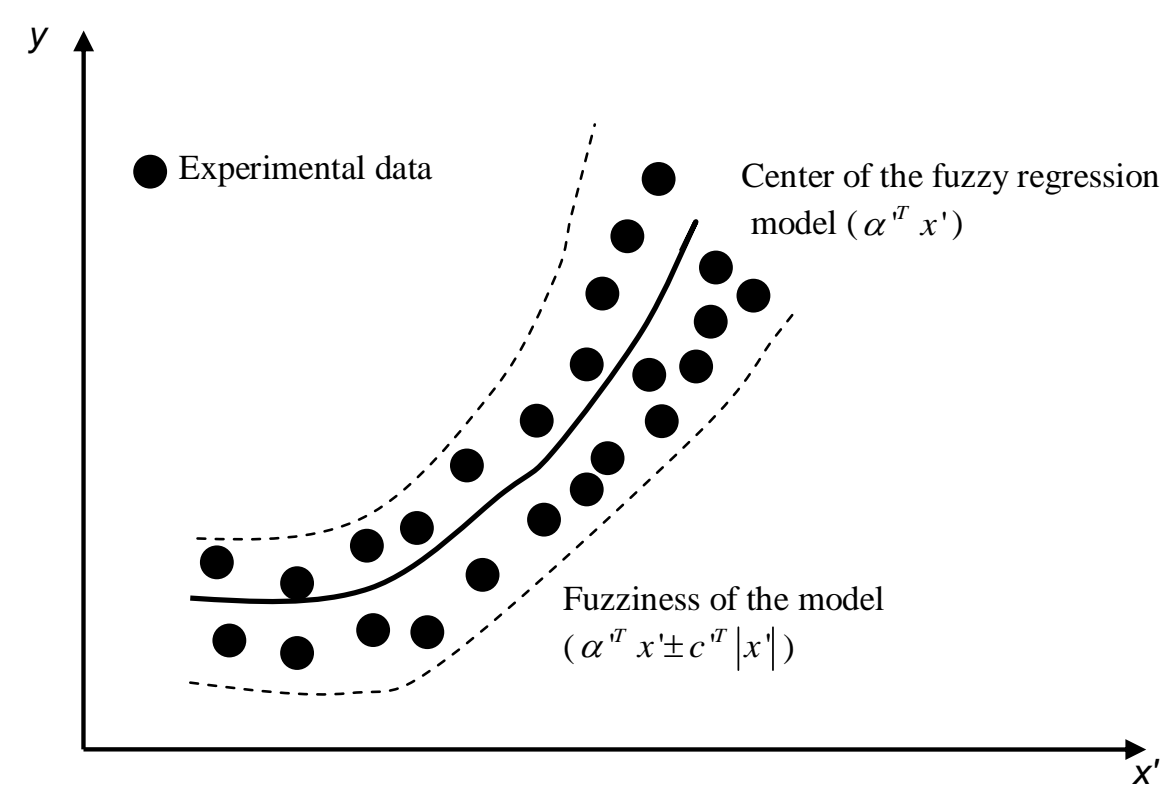

Figure 2 The fuzzy regression model for the epoxy dispensing process

\section{$3 \quad$ Hybrid fuzzy regression}

With reference to (3), since certain terms of (3) could be insignificant / redundant, they could be ignored such that a more parsimonious and adequate representation for the process could be obtained. The proposed hybrid fuzzy regression (HFR), which integrates genetic 
programming with the fuzzy regression, is proposed to determine the structure of the epoxy dispensing model and identify the significant fuzzy parameters of the model. The pseudocode of the HFR is shown in Figure 3, and the flowchart for each step described in the pseudocode is shown in Figure 4 in the appendix.

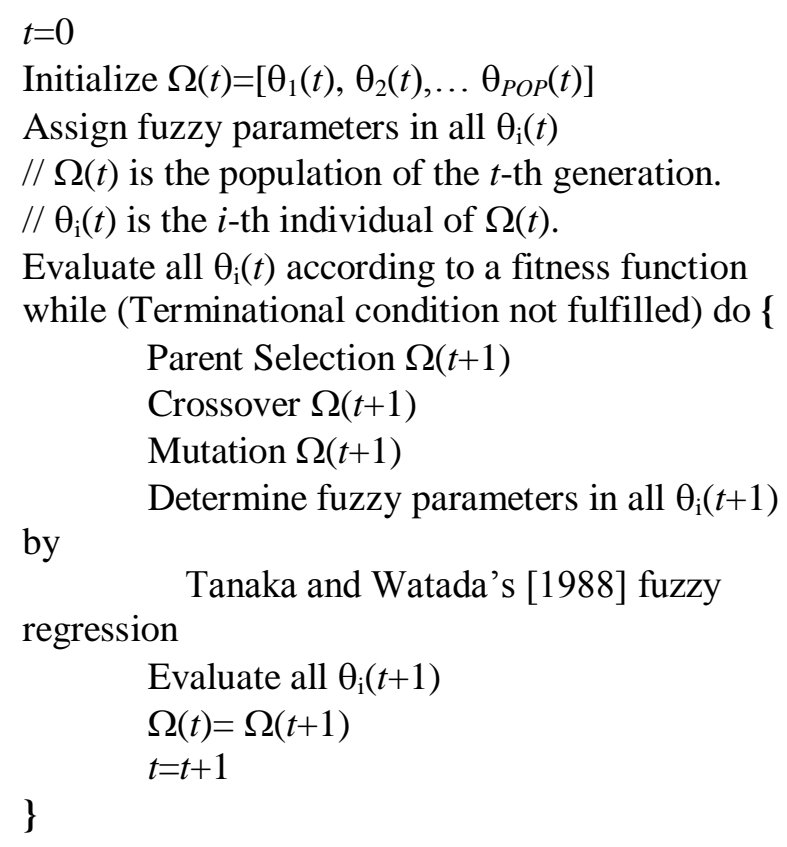

Figure 3 Pseudo code of the hybrid fuzzy regression

The HFR first starts with the creation of a random initial population $\Omega(t)$ with $P O P$ individuals $\theta_{i}(t)$, while $t=0$. Each individual $\theta_{i}(t)$ is in a form of a tree structure, that can be used to represent the structure of a fuzzy regression model as defined in (3). Then, the fuzzy parameters are assigned to each individual $\theta_{i}(t)$ by applying Tanaka and Watada's [21] fuzzy regression. All individuals are evaluated according to a defined fitness function which is aimed at evaluating the goodness-of-fitness of the fuzzy regression model. The parent selection process uses the goodness-of-fitness of each individual to determine the selection of potential individuals for performing crossover or mutation. Finally, the new individuals with the determined fuzzy parameters are evaluated using the fitness function to create a new population $\Omega(t+1)$. The process continues until the pre-defined termination condition is 
fulfilled. The major aspects of applying the HFR on modeling the epoxy dispensing process are described below:

\subsection{Model Representation}

HFR generates the potential solutions, which are represented as an epoxy dispensing model as shown in (3). One of the most popular methods to represent structures is the use of hierarchical trees composed of functions $F$ and terminals $T$ [11]. The fuzzy regression model (3) only contains the three arithmetic operations, + , - and $\times$; thus, $F$ is represented as $F=\{+$, $, \times\}$. The set of terminals $T=\{\boldsymbol{x}, \tilde{\mathbf{p}}\}$ contains the variable set $\boldsymbol{x}=\left\{x_{1}, x_{2}, x_{3}, x_{4}\right\}$ of the fuzzy

regression model and the fuzzy parameter set $\tilde{\mathbf{p}}=\left\{\tilde{p}_{0}, \tilde{p}_{1}, \tilde{p}_{2}, \ldots, \tilde{p}_{N_{N S}}\right\}$ of the fuzzy regression model, where $N_{N S}$ is the number of terms of the fuzzy regression model. For example, Figure 5 shows an example of a hierarchical tree for the epoxy dispensing model which expresses the following formulation:

$$
\left(x_{1} \times x_{1}\right)-\left(x_{2} \times x_{2}\right)+\left(x_{1} \times x_{2} \times x_{3}\right)
$$

which is equivalent to:

$$
x_{1}^{2}-x_{2}^{2}+x_{1} \cdot x_{2} \cdot x_{3}
$$

The fuzzy parameters set $\tilde{\mathbf{p}}=\left\{\tilde{p}_{0}, \tilde{p}_{1}, \tilde{p}_{2}, \tilde{p}_{3}\right\}$ is determined after identifying its structure from the hierarchical tree. In Figure 5, the number of fuzzy parameters of the epoxy dispensing model is 4 . Therefore, the completed fuzzy regression model can be represented as follows:

$$
\tilde{p}_{0} \cdot x_{0}+\tilde{p}_{1} \cdot x_{1}^{2}-\tilde{p}_{2} \cdot x_{2}^{2}+\tilde{p}_{3} \cdot x_{1} \cdot x_{2} \cdot x_{3}
$$




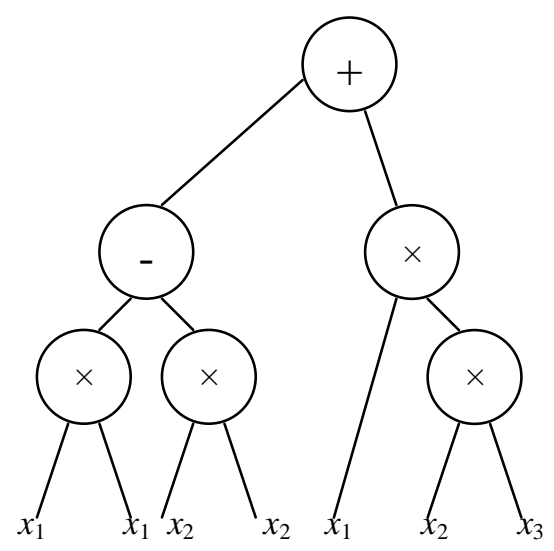

Figure 5 An example of a hierarchical tree for the epoxy dispensing model

It can also be represented by:

$\tilde{p}_{0} \cdot x_{0}+\tilde{p}_{1} \cdot x^{\prime}{ }_{1}-\tilde{p}_{2} \cdot x^{\prime}{ }_{2}+\tilde{p}_{3} \cdot x_{3}^{\prime}$,

where $x_{0}=1, x^{\prime}{ }_{1}=x_{1}^{2}, x^{\prime}{ }_{2}=x_{2}{ }^{2}$ and $x^{\prime}{ }_{3}=x_{1} \cdot x_{2} \cdot x_{3}$.

In this research, the fuzzy parameters are determined by Tanaka and Watada's [21] fuzzy regression, which is detailed in the appendix.

\subsection{Fitness function}

HFR evaluates the goodness-of-fitness of each individual by the fitness function, which is based on the mean absolute error (MAE), and can reflect the differences between the predicted values of the epoxy dispensing model and the actual values of the data sets. The MAE of the $j$-th individual can be calculated based on (4).

$$
M A E_{j}=100 \% \times \frac{1}{M} \sum_{k=1}^{M}\left|\frac{y(k)-F_{j}(x(k))}{y(k)}\right|,
$$

where $F_{j}$ is the epoxy dispensing model represented by the $j$-th individual; $(y(k), x(k))=\left(y(k),\left(x_{1}(k), x_{2}(k), x_{3}(k)\right)\right)$ is the $k$-th data set; and $M$ is the number of data sets used for developing the epoxy dispensing model.

(4) is commonly known as an indicator of modeling error of an epoxy dispensing model, reflecting how well the model can fit the training data sets. However, an epoxy 
dispensing model may contain many unnecessary and complex terms. A complex overparameterized epoxy dispensing model with a large number of parametrical terms reduces the transparency and interpretation of the epoxy dispensing model. To avoid the HFR from generating epoxy dispensing models that are too complex, a fitness function is designed to balance the tradeoff between the reduction of complexity and model accuracy. In this research, penalty terms are introduced into the fitness function of the HFR [22], and the fitness of the $j$-th individual is denoted as:

$$
\text { fitness }_{j}=\frac{1-M A E_{j}}{\left(1+\exp \left(c_{1}\left(L_{j}-c_{2}\right)\right)\right)}
$$

where fitness $_{j}$ is the fitness value, $L_{j}$ is the number of nodes of the $j$-th individual, and $c_{1}$ and $c_{2}$ are both the penalty terms.

\subsection{GP operations}

Like other evolutionary algorithms, the two main evolutionary operators in the HFR are crossover and mutation. For the crossover operation, one-point crossover with two parents is used. The crossover operation produces a pair of offspring that inherits the components of the epoxy dispensing models represented by two parents, and then the offspring is produced by exchanging the selected components of the two models. For example, two individuals, $\theta_{i}$ and $\theta_{j}$ represent the following models, and are shown in Figure 6(a):

$$
\theta_{i}=x_{3} * x_{1}-\left(x_{2} * x_{3}\right) \text { and } \theta_{j}=\left(x_{3} * x_{2}\right)+x_{2}
$$

After the crossover operation, the individuals are represented by the following two models and are shown in Figure 6b.

$$
\theta_{i}=x_{3} * x_{1}-\left(x_{2}\right) \text { and } \theta_{j}=\left(x_{3} * x_{2}\right)+\left(x_{2} * x_{3}\right)
$$

For mutation operation, point mutation is used. Mutation operation is performed by randomly selecting a node in the individual that is an internal or terminal node. Then, the 
associated sub-expression of the selected node is replaced with a randomly generated subexpression in the individual. For example, an individual $\theta_{i}$ (as shown in Figure 7) representing the following model is selected to be mutated:

$$
\theta_{i}=\left(x_{2}+x_{1}\right)+x_{1}
$$

The individual is mutated by replacing a plus in the node with a multiplier. After performing the mutation, the mutated individual became the following and is represented by Figure 7:

$$
\theta_{i}=\left(x_{2}+x_{1}\right) *\left(x_{1}\right)
$$

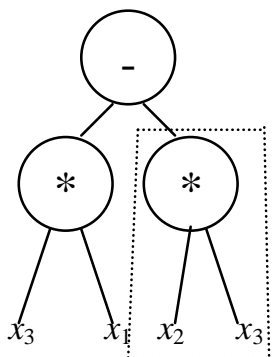

2-nd individual: $\theta_{j}$

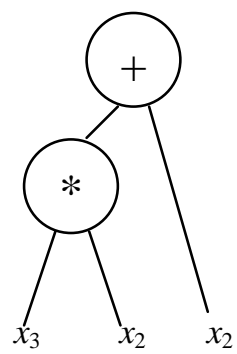

Figure 6(a) The individuals $\theta_{\mathrm{i}}$ and $\theta_{j}$ before performing crossover

1-st individual: $\theta_{i}$,

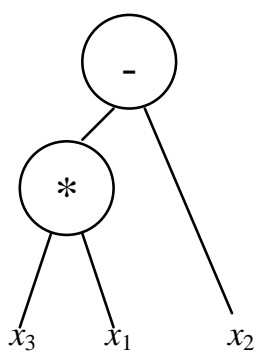

2-nd individual: $\theta_{j}$ '

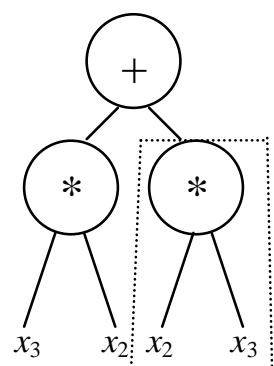

Figure 6(b) The individuals $\theta_{\mathrm{i}}$ ' and $\theta_{\mathrm{j}}$ ' after performing crossover 
The individual $\theta_{k}$ before the mutation

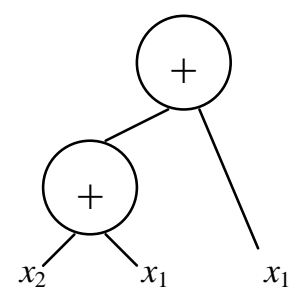

The individual $\theta_{k}$ ' before the mutation

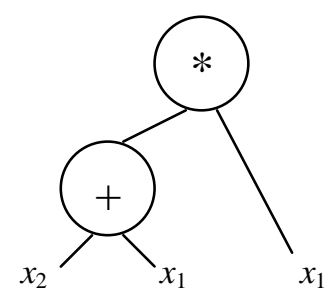

Figure 7 Individual produced by the mutation

After the crossover and mutation operations, individuals from the current population with relatively better fitness, that can produce better epoxy dispensing model, are selected to serve as parents for the next generation. The roulette-wheel approach, which is among the most common selection methods used for selecting individuals to perform reproduction operations in evolutionary algorithms [23], is used for selection of individuals. After the selection, the population is evolved and improved iteratively until the pre-defined number of generations is reached. Otherwise, the HFR goes to the next evolutionary iteration.

\section{$4 \quad$ Model development}

In this research, 12 experiments were carried out based on a full factorial design with two levels of compressed air pressure $\left(x_{1}\right)$, two levels of pump motor speed $\left(x_{2}\right)$, and three levels of height between the substrate and the needle $\left(x_{3}\right)$. In the experiments, the substrate temperature and the needle temperature were set to $60{ }^{\circ} \mathrm{C}$ and $40{ }^{\circ} \mathrm{C}$, respectively. The experimental plan and results are shown in Table 1 . The values as shown within the brackets in the table are the normalized ones, which range from 0 to 1 . As suggested by the company supporting this research, it is practical that this small amount of experimental data is only available. Usually, it is impossible to collect a large amount of experimental results to develop the process models. Even though the process models with better accuracies are more likely to be generated, when larger amount of experimental data is used, larger amount of 
time is required to conduct the experiments. Therefore, this small amount of experimental data is used to develop the process models for the epoxy dispensing process, in order to evaluate whether appropriate process models with reasonable accuracies can be generated by the proposed HFR.

Table 1 Experimental data sets of the epoxy dispensing process

\begin{tabular}{|c|c|c|c|c|c|}
\hline $\begin{array}{c}\text { Run no. } \\
K\end{array}$ & $\begin{array}{c}\text { Pressure } \\
(\mathrm{psi}) \\
x_{1 k}\end{array}$ & $\begin{array}{c}\text { Speed (rpm) } \\
x_{2 k}\end{array}$ & $\begin{array}{c}\text { Height (step) } \\
x_{3 k}\end{array}$ & $\begin{array}{c}\text { Encap. } \\
\text { weight (mg) } \\
y_{k}\end{array}$ & $\begin{array}{c}\text { Encap. } \\
\text { thickness } \\
(\mathrm{mm}) \\
z_{k}\end{array}$ \\
\hline 1 & $1(0)$ & $400(0)$ & $500(0)$ & 75.6 & 0.64 \\
\hline 2 & $1(0)$ & $400(0)$ & $1000(0.5)$ & 74.6 & 0.59 \\
\hline 3 & $1(0)$ & $400(0)$ & $1500(1)$ & 72.3 & 0.58 \\
\hline 4 & $1(0)$ & $1000(1)$ & $500(0)$ & 31 & 0.44 \\
\hline 5 & $1(0)$ & $1000(1)$ & $1000(0.5)$ & 31.1 & 0.42 \\
\hline 6 & $1(0)$ & $1000(1)$ & $1500(1)$ & 31.8 & 0.44 \\
\hline 7 & $4(1)$ & $400(0)$ & $500(0)$ & 114.3 & 0.74 \\
\hline 8 & $4(1)$ & $400(0)$ & $1000(0.5)$ & 111.9 & 0.72 \\
\hline 9 & $4(1)$ & $400(0)$ & $1500(1)$ & 113.8 & 0.72 \\
\hline 10 & $4(1)$ & $1000(1)$ & $500(0)$ & 51.4 & 0.50 \\
\hline 11 & $4(1)$ & $1000(1)$ & $1000(0.5)$ & 53.1 & 0.53 \\
\hline 12 & $4(1)$ & $1000(1)$ & $1500(1)$ & 55.3 & 0.53 \\
\hline
\end{tabular}

Using the 12 normalized experimental data sets and the experimental results as shown in Figure 1, the following statistical regression models, (6) and (7) respectively, for encapsulation weight and encapsulation thickness were generated by the statistical regression (SR) based on the Matlab statistical toolbox:

$$
y=78.3541+30.5667 \cdot x_{1}-51.4667 \cdot x_{2}+0.2250 \cdot x_{3}
$$

The $\mathrm{R}^{2}$ value of model (6) is $97.8 \%$ and its training error with respect to the data sets in Table 1 is $7.8793 \%$.

$$
z=0.6188+0.1050 \cdot x_{1}-0.1883 \cdot x_{2}+0.0125 \cdot x_{3}
$$

The $\mathrm{R}^{2}$ value of model (7) is $97.3 \%$ and its modeling error is $2.6820 \%$.

Using the same data sets, the fuzzy linear regression models for encapsulation weight and encapsulation thickness were generated based on the approach of fuzzy linear regression (FR) [12] as shown in (8) and (9), respectively. 


$$
\begin{aligned}
& y=(100.4256,2.4537)+(10.3556,0.7097) x_{1} \\
& +(-0.0833,0.0014) x_{2}+(-0.0005,0.0004) x_{3} \\
& z=(0.7222,0.0196)+(0.0309,0.0016) x_{1} \\
& +(-0.0003,0.0000) x_{2}+(-0.0000,0.0000) x_{3}
\end{aligned}
$$

Their training errors are $7.8474 \%$ and $3.2624 \%$, respectively.

It can be found that the training errors obtained by statistical regression models given in equations (6) and (7) are similar to the those obtained by the fuzzy linear regression models given in (8) and (9). The training error obtained by the fuzzy linear regression for encapsulation weight is only slightly better than those obtained by the statistical regression. Also, the training error obtained by the statistical regression for encapsulation thickness is almost the same as those obtained by the fuzzy linear regression. However, more information can be provided by the models which are generated based on the fuzzy linear regression. The fuzziness of each parameter can be indicated by the fuzzy linear regression models, which are represented by equations (8) and (9). Both equations (8) and (9) show that the fuzziness produced by the process parameter namely Pressure $\left(x_{1}\right)$ is the higher than those of the process parameters namely Speed $\left(x_{2}\right)$ and Height $\left(x_{3}\right)$. However, this fuzzy information cannot be indicated by the statistical regression models, which is one of the limitations of using statistical regression.

Then, the same experimental data sets as shown in Table 1 were used to develop the HFR models for the epoxy dispensing process using Matlab. Madar et al. [18] found that the GP parameters given in Table 2 are able to find good solutions for various problems. As this paper focus on the development of the mechanisms of the HFR, these GP parameters are used in the HFR. 
Table 2 GP parameters setting of the HFR

\begin{tabular}{|c|c|}
\hline Population size & 50 \\
\hline Maximum number of evaluated individuals & 5000 \\
\hline Generation gap & 0.9 \\
\hline Probability of crossover & 0.5 \\
\hline Probability of mutation & 0.5 \\
\hline
\end{tabular}

Since the HFR is a stochastic method, different results could be obtained from different runs. To evaluate its overall performance, 30 runs were carried out. The best HFR models for encapsulation weight and encapsulation thickness with the smallest modeling errors among the 30 runs are shown in (10) and (11), respectively.

$$
\begin{aligned}
& y=(73.8000,18.0000)+(39.2634,0.0000) \cdot x_{1}+(-42.6526,0.0000) \cdot x_{2} \\
& +(0.3000,0.0001) \cdot x_{3}+(-17.2108,0.0001) \cdot x_{1} \cdot x_{2} \\
& z=(0.6288,0.1125)+(0.1019,0.0000) \cdot x_{1}+(-0.1950,0.0002) \cdot x_{2} \\
& +(-0.0055,0.0000) \cdot x_{3}+(-0.0394,0.0000) \cdot x_{1} \cdot x_{2} \\
& +(0.0500,0.0007) \cdot x_{1} \cdot x_{3}+(0.0500,0.0007) \cdot x_{1} \cdot x_{2}
\end{aligned}
$$

The training errors for encapsulation weight and encapsulation thickness are $1.4857 \%$ and $1.8074 \%$, respectively.

It can be seen that interaction and high order terms exist in the models (10) and (11), which are generated based on the HFR, but do not exist in models (6), (7), (8) and (9), which are developed based on the other two methods. Also, equation (10) shows that the parameter $x_{1}$, and the interaction between parameters $x_{1}$ and $x_{2}$, induce most significant fuzziness to the encapsulation weight. Equation (11) shows that the interaction between parameter $x_{1}$ and $\mathrm{x}_{3}$, and the interaction between parameters $x_{1}$ and $x_{2}$, induce most significant fuzziness to the encapsulation thickness. 
To present the testing results more clearly, Table 3 summarizes all the training errors of the process models developed based on statistical regression (SR), fuzzy linear regression (FLR) and the proposed HFR. In Table 3, it can be found that the training errors for both encapsulation weight and encapsulation thickness obtained by the proposed HFR are the smallest, comparing with those obtained by the statistical regression and the fuzzy linear regression. It can be explained by the fact that interaction terms or high order terms can be generated by the HFR, while both statistical regression and fuzzy linear regression can only generate linear terms. Therefore, both high order terms and nonlinear relationships of process parameters are more likely to be addressed, based on the process models generated by the HFR. Hence, the capability of the HFR for fitting the experimental data is better than those for the statistical regression and fuzzy linear regression, and the training errors obtained by the HFR is smaller than those obtained by both fuzzy linear regression and statistical regression.

Table 3 Comparisons of the training results

\begin{tabular}{|c|c|c|c|}
\hline & SR & FR & HFR \\
\hline Training error of & 7.8793 & 8.2177 & 1.4857 \\
encapsulation weight (\%) & & & \\
\hline Training error of & 2.6820 & 3.1832 & 1.8074 \\
encapsulation thickness $(\%)$ & & & \\
\hline
\end{tabular}

Cross-validation [24] is used to evaluate the effectiveness of the HFR as compared to the statistical regression and fuzzy linear regression in modeling the epoxy dispensing process. One round of cross-validation involves partitioning the 12 data sets into 2 subsets. It performs the analysis on one subset namely the cross-training set, and validates the analysis on the other subset namely the cross-validation set. Here 10 of the 12 data sets are randomly selected as the cross-training set to develop the model, while the remaining 2 data sets are 
used as the cross-validation set to test the models. The two measures, training error regarding the cross-training set and testing error regarding cross-validation set, are used to evaluate the effectiveness of the HFR. The training error is used to reflect how well a developed model can fit the cross-training sets for developing models. The testing error is used to reflect how well the developed model can predict a response, based on the cross-validation set. As multiple rounds of cross-validation are performed using different partitions, and the validation results are averaged over the rounds, the variability in term of both modeling and testing errors can be reduced.

For the encapsulation weight and encapsulation thickness, the 12 modeling errors based on the three methods, statistical regression, fuzzy linear regression and HFR, are shown in Figure 8 and Figure 9, respectively. The $x$-axis of the figures indicates the cross-validation sets used for testing. For example, $(2,9)$ means that the $2^{\text {nd }}$ and $9^{\text {th }}$ data sets were used for testing, and the rest of the data sets were used for model building. It can be seen from the figures that the line indicating the training errors for the HFR is lower than those for the statistical regression and fuzzy linear regression. Therefore, HFR can yield the smallest training errors in modeling for both the encapsulation weight $y$ and encapsulation thickness $z$, compared with those obtained by both statistical regression (SR) and fuzzy linear regression (FR). Figure 10 and Figure 11 show the testing errors based on the methods for the encapsulation weight $y$ and the encapsulation thickness $z$, respectively. From the figures, it can be found that the line indicating the testing errors for the HFR is the lowest, compared with those of the statistical regression and fuzzy linear regression. Therefore, the models based on the HFR can yield the smallest testing errors in both the quality characteristics, compared with those obtained by both statistical regression and fuzzy linear regression. Therefore, these results suggest that HFR not only has better capability for fitting the training data, but also has higher generalization capability in estimating both the encapsulation weight $y$ and encapsulation thickness $z$ than the other five algorithms in general. 


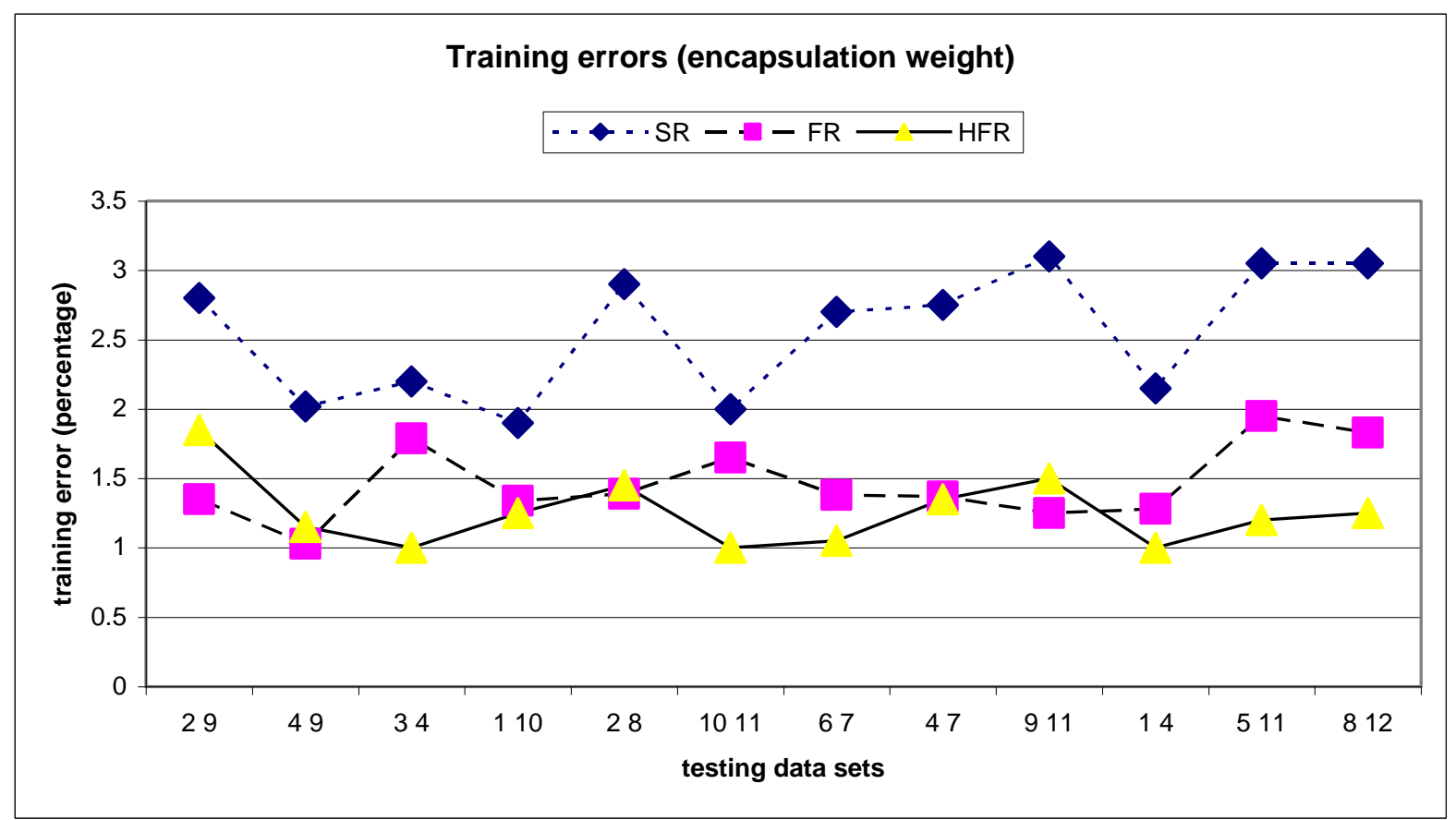

Figure 8 Training errors for encapsulation weight

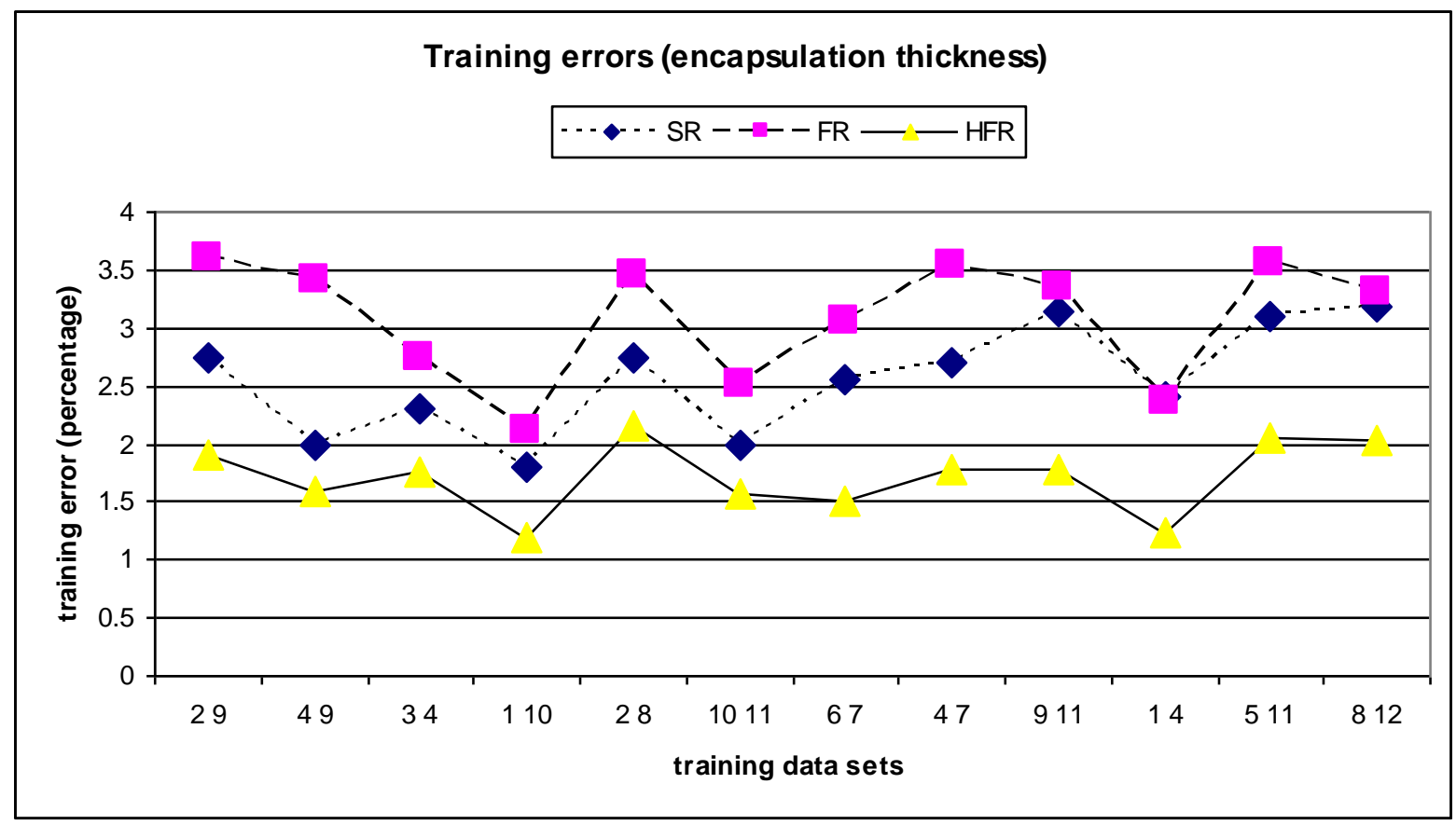

Figure 9 Training errors for encapsulation thickness 


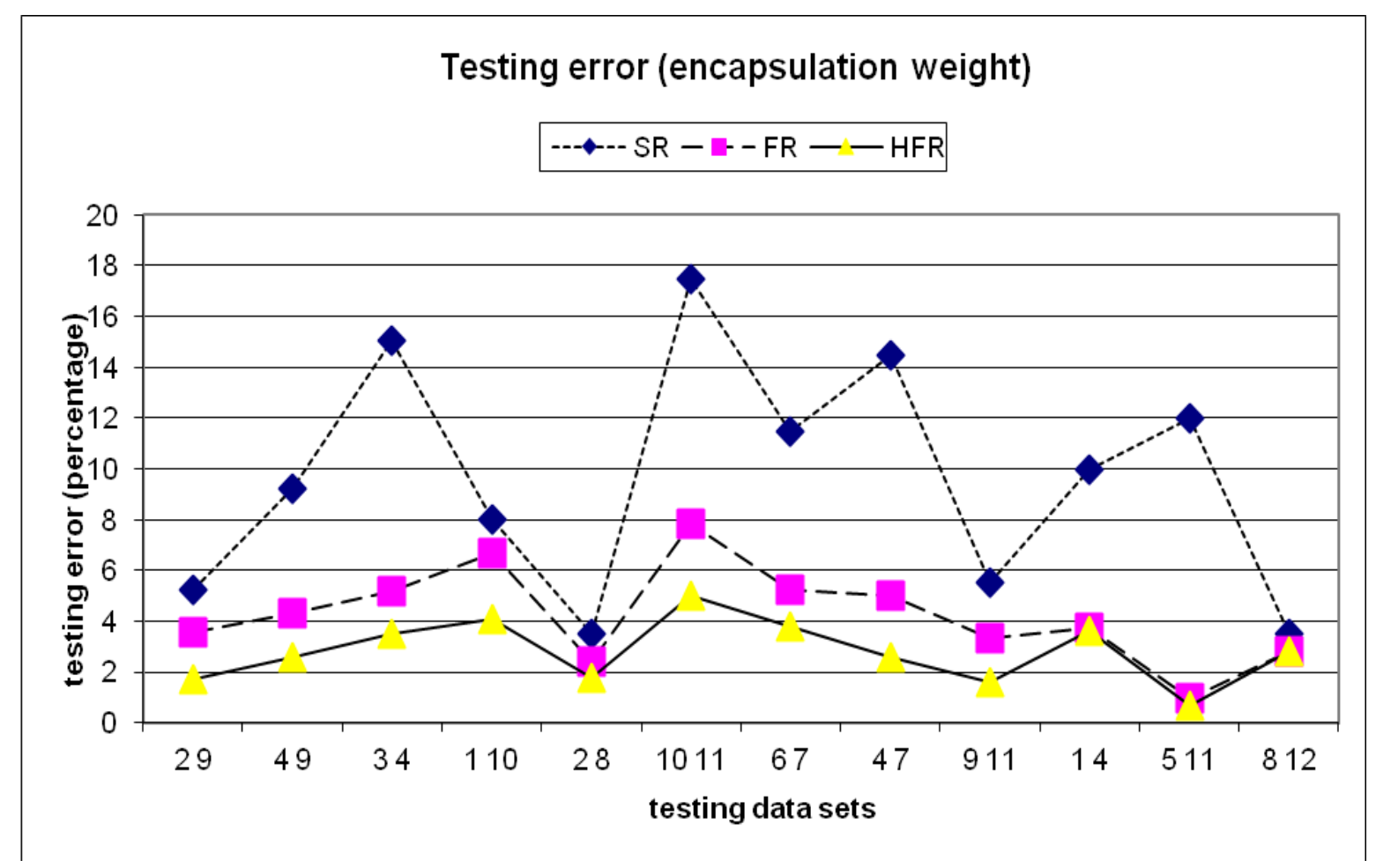

Figure 10 Testing errors for encapsulation weight

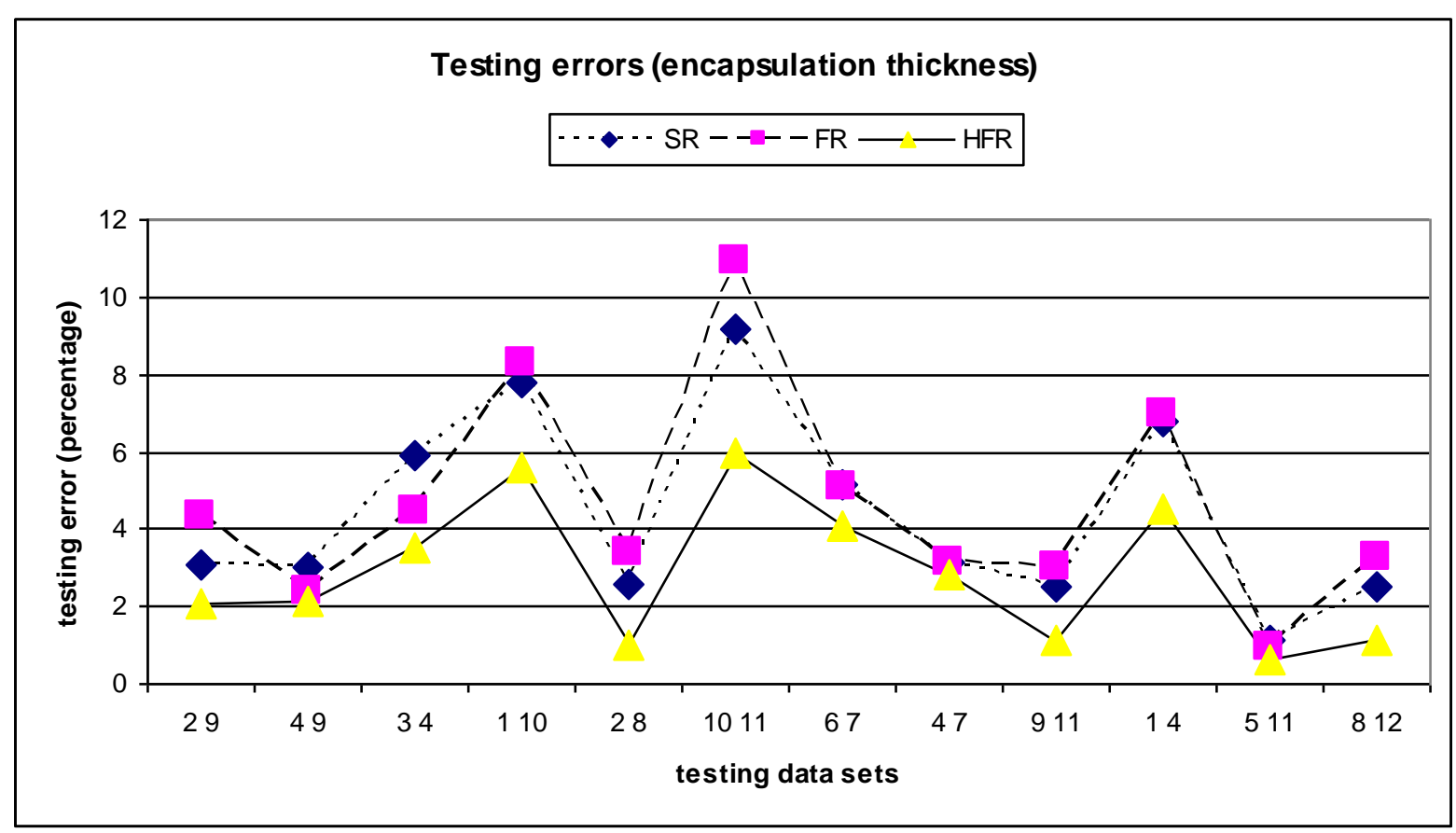

Figure 11 Testing errors for encapsulation thickness

The means of the 12 testing errors of the three methods for the encapsulation weight $y$ and encapsulation thickness $z$ are shown in Table 4, from which it can be found that the HFR 
based models can yield the smallest testing errors. Also table 4 shows that the mean testing error obtained for the encapsulation weight is smaller than those obtained for the encapsulation thickness. It can be explained by two reasons: 1) encapsulation thickness is more difficult to be measured or calibrated than the encapsulation weight. Measurement errors for the encapsulation thickness are generally higher than those for the encapsulation weight. 2) Also, it is more difficult to control the shape and the volume of the epoxy by adjusting the process parameters than to control the encapsulation weight, so encapsulation thickness is more difficult to be controlled. Therefore, the models developed for the encapsulation thickness are more likely to produce higher errors than those for the encapsulation weight.

Table 4 Means of testing errors for encapsulation weight and encapsulation thickness

\begin{tabular}{|l|c|c|}
\hline & $\begin{array}{l}\text { Encapsulation weight } \\
\text { (mean of testing errors \%) }\end{array}$ & $\begin{array}{l}\text { Encapsulation thickness } \\
\text { (mean of testing errors \%) }\end{array}$ \\
\hline SR & 2.5441 & 4.0987 \\
\hline FR & 3.0161 & 4.2219 \\
\hline HFR & 1.7406 & 2.9252 \\
\hline
\end{tabular}

\section{$5 \quad$ Conclusion}

In this paper, a hybrid fuzzy regression (HFR) approach integrating fuzzy regression with genetic programming has been proposed to model the epoxy dispensing process for microchip encapsulation. Experiments based on full factorial design were conducted in which the process parameters of compressed air pressure, pump motor speed, distance between substrate and needle, and two quality characteristic, encapsulation weight and encapsulation thickness, were involved. In the HFR, the general outcomes of genetic programming were used to construct the structures of the epoxy dispensing models based on a tree 
representation, and where the fuzzy regression algorithm was then employed to estimate the contributions and the fuzziness of the branches of the tree, so as to identify the fuzzy parameter of each term of the epoxy dispensing model. Since interaction and high order terms can be introduced in the branches of the tree using the genetic programming, a non-linear fuzzy regression model with interaction terms was developed based on the HFR approach.

To evaluate the performance of the proposed HFR approach, it has been applied to modeling the epoxy dispensing process, which is highly nonlinear process, due to the complex epoxy characteristics. Also, the results obtained by the HFR have also been compared with the other commonly used explicit modeling methods, including statistical regression and fuzzy linear regression. The result shows that the smallest training errors can be achieved by the HFR. This results indicate that the HFR is more capable to fit the data sets than the other two tested methods (i.e. statistical regression and fuzzy linear regression). In addition, a comparison of the validation results shows that smallest training errors and testing errors can be obtained by the HFR, compared with those obtained by statistical regression and fuzzy linear regression. The achievement of better results can be explained by the reason that interaction terms or high-order terms can be generated by HFR, but the other two tested methods ignore them. The results of the comparisons indicate that the HFR outperformed statistical regression and fuzzy regression in modeling the epoxy dispensing process in terms of training errors and testing errors.

Further research will involve the investigation of using different fuzzy membership functions on the HFR, in order to optimize the performance of the HFR. Also, physical experiments will be conducted, in order to further validate the effectiveness of the models developed by the HFR. 


\section{Acknowledgement}

The work described in this paper was supported substantially by a grant from the Department of Industrial and Systems Engineering, The Hong Kong Polytechnic University.

\section{References}

[1] Chen D.X. (2002) Modeling and off-line control of fluid dispensing for electronics packaging. $\mathrm{PhD}$ thesis, The University of Saskatchewan

[2] Chen X.B., Ke H. (2006) Effect of fluid properties on dispensing processes for electronic packaging. IEEE Transactions on Electronic Packaging Manufacturing 29(2): 75-82

[3] Li H.X., Tso S.K., Deng H. (2001) A concept approach to integrate design and control for the epoxy dispensing process. International Journal of Advanced Manufacturing Technology 17: 677-682

[4] Hong Y.P., Li H.X. (2003) Comparative study of fluid dispensing modeling. IEEE Transactions on Electronic Packaging Manufacturing 26(4): 273-280

[5] Li J., Deng G. (2004) Technology development and basic theory study of fluid dispensing - a review. Proceedings of the sixth IEEE Components, Packaging and Manufacturing Technology Conference 198-205

[6] Chen X.B. (2009) Modeling and control of fluid dispensing processes: a state-of-the-art review. International Journal of Advanced Manufacturing Technology 43: 276-286

[7] Kwong C.K., Chan K.Y., Wong H. (2007) An empirical approach to modeling fluid dispensing for electronic packaging. International Journal of Advanced Manufacturing Technology 34(1-2): 111-121

[8] Kwong C.K., Chan K.Y., Wong H. (2008) Takagi-Sugeno neural fuzzy modeling approach to fluid dispensing for electronic packaging. Expert Systems with Applications 34(3): 2111-2119 
[9] Takagi T., Sugeno M. (1985) Fuzzy identification of systems and its application to modeling and control. IEEE Transactions on Systems, Man and Cybernetics 15(1): 116132

[10] Azadeh A., Seraj O., Saberi M. (2011) An integrated fuzzy regression - analysis of variance algorithm for improvement of electricity consumption estimation in uncertain environments. International Journal of Advanced Manufacturing Technology 53: 645660

[11] Sener Z., Karsak E.E. (2010) A decision model for setting target levels in quality function development using nonlinear programming-based fuzzy regression and optimization. International Journal of Advanced Manufacturing Technology 48: 11731184

[12] ] Ip K. W., Kwong C. K., Bai H., Tsim Y. C. (2003) Process modelling of epoxy dispensing for microchip encapsulation using fuzzy linear regression with fuzzy intervals. International Journal of Advanced Manufacturing Technology 22: 417-423

[13] Koza J. (1992) Genetic Programming: On the Programming of Computers by Means of Natural Evolution. MIT Press: Cambridge

[14] Koza J. (1994) Genetic Programming II: automatic discovery of reusable programs. MIT Press

[15] Lakshminarayanan S., Fujii H., Grosman B., Dassau E., Lewin D.R. (2000) New product design via analysis of historical databases. Computers and Chemical Engineering 24: $671-676$

[16] Brezocnik M., Gusel L. (2004) Predicting stress distribution in cold formed material with genetic programming. International Journal of Advanced Manufacturing Technology 23: 467-474 
[17] Kok M., Kanca E., Eyercioglu O. (2010) Prediction of surface roughness in abrasive waterjet machining of particle reinforced MMCs using genetic expression programming. International Journal of Advanced Manufacturing Technology

[18] Madar J., Abonyi J., Szeifert F. (2005) Genetic programming for the identification of nonlinear input - output models. Industrial and Engineering Chemistry Research 44: $3178-3186$

[19] Kwong C.K., Chan K.Y. (2005) Process modeling and optimization of epoxy dispensing using volumetric pump. A Progress Report to ASM Assembly Automation Ltd.

[20] Gabor D., Wildes W., Woodcock R. (1961) A Universal non-linear filter, predictor and simulator which optimizes itself by a learning process. Proceedings of IEE 108B: 422438

[21] Tanaka H., Watada J. (1988) Possibilistic linear systems and their application to the linear regression model. Fuzzy Sets and Systems 272: 275-289

[22] Willis M.J., Hiden H., Hinchliffe M., McKay B., Barton G.W. (1997) Systems modeling using genetic programming. Computers and Chemical Engineering 21: 1161-1166.

[23] Goldberg D.E. (1989) Genetic Algorithms in Search, Optimization and Machine Learning. Addison Wesley.

[24] Stone M. (1974) Cross validation choice and assessment of statistical predictions, Journal of the Royal Statistical Society 36: 111-147.

[25] Tanaka H., Uejima S., Asai K. (1982) Linear regression analysis with fuzzy model. IEEE Transactions on Systems, Man, and Cybernetics 12: 903-907 


\section{Appendix}

Tanaka et al. [25] formulated the fuzzy regression problem as the following linear programming problem:

$$
\text { Minimize } \quad J=\sum_{j=1}^{N_{N R}}\left(c_{0}^{\prime}+c^{\prime}{ }_{j} \sum_{i=1}^{M}\left|x^{\prime}{ }_{j}(i)\right|\right)
$$

where $M$ is the number data sets used for training the epoxy dispensing model, and $x^{\prime}{ }_{j}(i)$ is the $i$-th data set with respect to the $j$-th transformed variable of the epoxy dispensing model, subject to:

$$
\begin{aligned}
& \left(\alpha_{0}^{\prime}+\sum_{j=1}^{N_{N R}} \alpha_{j}^{\prime} x_{j}^{\prime}(i)\right)+(1-h)\left(c_{0}^{\prime}+\sum_{j=1}^{N_{N R}} c^{\prime}{ }_{j} \mid x^{\prime}{ }_{j}(i)\right) \geq y(i) \\
& \left(\alpha_{0}^{\prime}+\sum_{j=1}^{N_{N R}} \alpha^{\prime}{ }_{j} x^{\prime}{ }_{j}(i)\right)+(1-h)\left(c_{0}^{\prime}+\sum_{j=1}^{N_{N R}} c^{\prime}{ }_{j} \mid x^{\prime}{ }_{j}(i)\right) \leq y(i) \\
& c_{j} \geq 0, \quad \alpha_{j} \in R, \text { for all } i, \\
& 0 \leq h \leq 1, i=1,2, \cdots M, j=1,2, \cdots N_{N R} .
\end{aligned}
$$

$J$ in (18) is the total fuzziness of the epoxy dispensing model. The value of $h$ in (19) and (20) is between 0 and 1 . It is referred to as a fitting degree of the epoxy dispensing model to the given data sets, and is subjectively chosen by decision makers. Constraints (19) and (20) restrict that the observation of the $i$-th data set $y(i)$ has at least $h$ degree of belonging to $\tilde{y}(i)$ as $\mu_{\tilde{y}(i)}(y(i)) \geq h(i=1,2, \cdots, M)$. Therefore, the objective of solving the linear programming problem (18-22) was to determine the fuzzy nonlinear parameters $\tilde{A}_{j}^{\prime}=\left(c^{\prime}{ }_{j}, \alpha^{\prime}{ }_{j}\right)$ such that the total vagueness $J$ is minimized subject to $\mu_{\tilde{y}(i)}(y(i)) \geq h(i=1,2, \cdots, M)$. Therefore, the intervals of the epoxy dispensing process derived from Tanaka et al [25]'s approach were determined by all the collected data sets and the value $h$. With the advice from the company supporting this research [18], $h=0.1$ was used. 


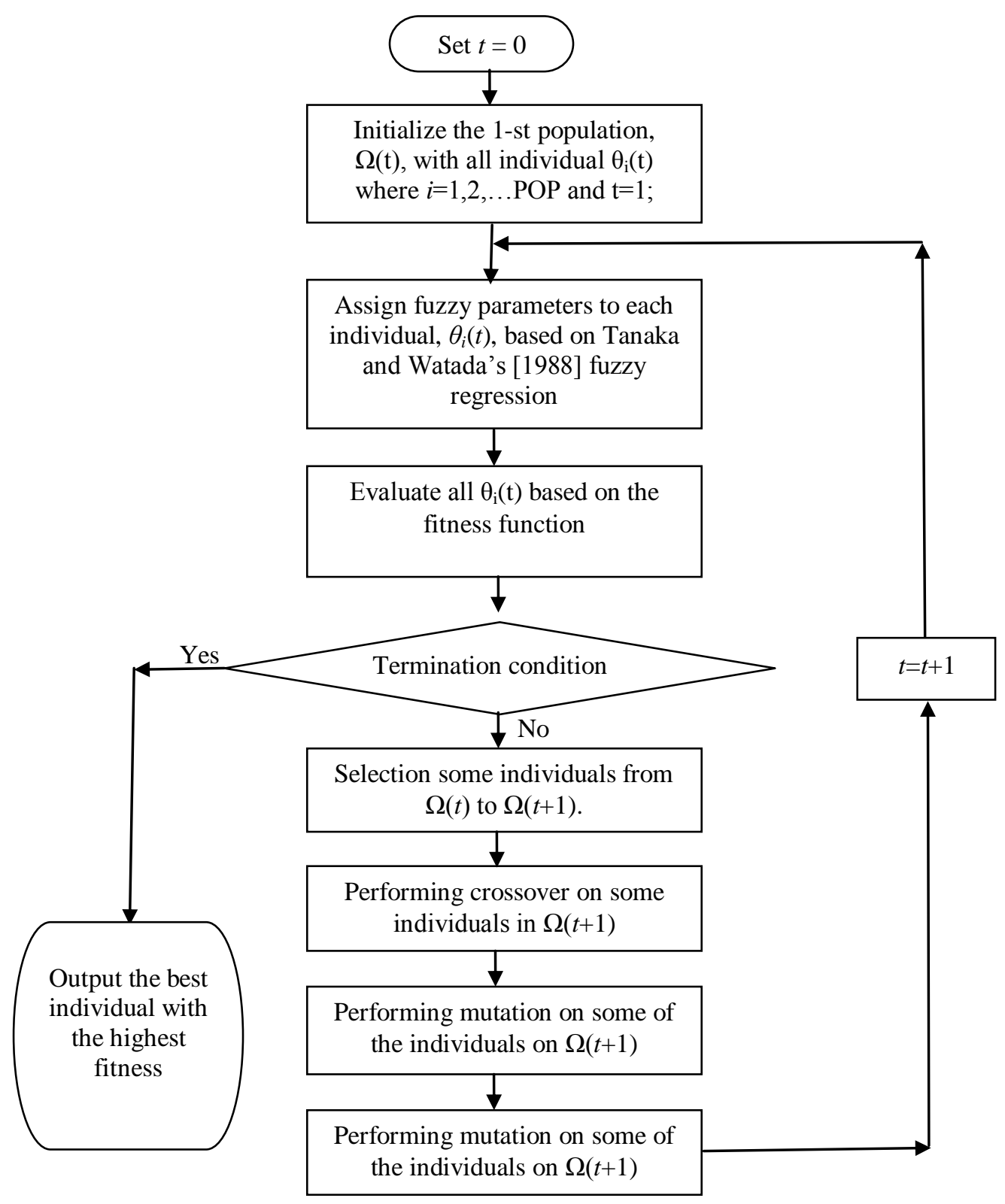

Figure 4 The flowchart of the hybrid fuzzy regression (HFR) 